\title{
Is NAT The Best Screening Modality Of Infectious Disease Seroprevalence in Modern Blood Banking? A Comparative Analysis between Chemiluminescence and ID-NAT in a Tertiary Care Centre
}

\section{Sumbul W' ${ }^{1}$, Shankul J ${ }^{2}$, Kafil $\mathrm{A}^{1 *}$ and Arif $\mathrm{SH}^{1}$}

${ }^{1}$ Department of Pathology, Jawaharlal Nehru Medical College, India

${ }^{2}$ Department of Preventive and Social Medicine, Jawaharlal Nehru Medical College, India

*Corresponding author: Kafil Akhtar, Professor, Department of Pathology, Jawaharlal Nehru Medical College, Aligarh Muslim University, Aligarh, U.P, India, Email: drkafilakhtar@gmail. com

\section{Research Article}

Volume 4 Issue 1

Received Date: January 06, 2020

Published Date: January 17, 2020

DOI: $10.23880 /$ hij-16000151

\section{Abstract}

Aims and Objectives: To determine the sero-prevalence of HIV, HBV and HCV infections in blood donors by dual testing strategy using high sensitivity screening assays like enhanced chemiluminescence technology and nucleic acid testing (IDNAT).

Introduction: Safe transfusion of blood and blood components saves millions of lives, but unsafe transfusion practices put millions of people at risk of transfusion transmittable infections.

Material and Methods: Blood was collected from voluntary and replacement donors and all serum samples were screened for anti HIV 1 and 2 antibody, anti HCV antibody and HBsAg by chemiluminescence and ID-NAT and the results were analysed statistically.

Results: Majority of the donors were in the 3rd decade of life,13742 (59.5\%); followed by in 4th decade of life, 4547 (19.7\%). There were 22303(96.6\%) males as compared to 771(3.3\%) female donors, with male-female ratio of 28.9:1. Replacement donations were 21392 (92.7\%) and voluntary donations were 1682 (7.2\%). The seroprevalence of HIV was 29(0.1\%), HCV was $160(0.6 \%)$, HbsAg was 508(2.2\%) and 22377(96.9\%) donors were non-reactive by chemiluminescence. The seroprevalence of HIV was $18(0.07 \%)$, HCV was $137(0.5 \%)$, HbsAg was 598(2.5\%) and 22321(96.7\%) donors were non-reactive by ID-NAT. The sensitivity, specificity and diagnostic accuracy by chemiluminescence was $97.7 \%, 95.5 \%$ and $97.0 \%$ respectively and the sensitivity, specificity and diagnostic accuracy by ID-NAT was $99.2 \%, 96.7 \%$ and $99.8 \%$ respectively.

Conclusion: ID-NAT is superior to Chemiluminescence in detecting infectious blood units in all phases of infection and enhances the safety of the blood and component transfusion.

Keywords: Blood Donors, Seroprevalence, Infectious Diseases, ID-NAT, Chemiluminescence 


\section{Haematology International Journal}

Abbreviations: TTIs: Transfusion Transmittable Infections; CIA: Chemiluminescent Immunoassay; ELISA: Enzyme-Linked Immüno-Sorbant Assay; RIBA: Recombinant Immunoblot Assay; NAT: Nucleic Acid Test; ID-NAT: Chemiluminescence Technology and Nucleic Acid Testing.

\section{Introduction}

Blood safety is major concern globally going by the increasing incidence of transfusion transmittable infections (TTIs). Safe transfusion of blood and blood components saves millions of lives, but unsafe transfusion practices put millions of people at risk of TTIs. Blood is one of the major sources of transmission of infectious diseases, viz. HIV, HBV, HCV, syphilis, and many other infections in India. With an estimated population of 1.21 billion, India has the world's third largest population suffering from HIV/AIDS. The estimated adult HIV prevalence was $0.31 \%$ in 2009 [1]. India has intermediate endemicity of hepatitis B with HBsAg prevalence of $2-10 \%$ among the study population. It has been estimated that up to 40 million people out of the 350 million hepatitis B chronic carriers worldwide arise in India [2]. In India, there are about 12-13 million HCV carriers and modeling data predict that the burden of disease could soon increase substantially [3].

Parenteral transmission through blood transfusion and infected needles and syringes remain the most significant route of transmission for infectious diseases in our country. Blood transfusion is an effective mode of transmission as it allows a large quantum of infective virions into the susceptible patient. In developed countries, numerous corrective measures have reduced the spread of infection through this route. In India, mandatory screening for HCV was introduced in 2002. Many of the more recent blood donor studies report prevalence of $<1.0 \%$, indicating that increased screening and education of donors is working. Replacement donors typically have higher HCV infection rates than voluntary donors [4].

Antibody detection tests like chemiluminescent immunoassay (CIA) and enzyme-linked immüno-sorbant assay (ELISA) tests are the most frequently serological tests which are used for diagnosis of HCV infection [5]. These serological tests are rapid, easy and cheap but false positive results frequently have been observed especially for antibody tests [6]. CDC has recommended that a person can be considered to have serologic evidence of HCV infection only after an anti-HCV screening-test-positive result has been verified by a more specific serologic test, e.g., the recombinant immunoblot assay (RIBA) or a nucleic acid test (NAT) [7].

With increasing voluntary blood donation and still prevalent infectious diseases in donors, we need to augment transfusion transmitted infection testing before use. Nucleic acid testing (NAT) blood screening for key transfusiontransmitted infections (TTIs) was originally intended to complement serological screening for detection of donations infectious for those viruses. The main advantage of NAT screening is interdiction of new incident cases during the window period infections and identification of occult hepatitis B carrier status which can potentially be infectious [8]. This tool could provide the next large step in improving the safety of blood in India and adding to the epidemiological database of incidence and prevalence of the viral infections [9], where 6 million units of blood are collected annually [10].

The objective of this study was to determine the seroprevalence of HIV, HBV and HCV infections in blood donors by dual testing strategy using high sensitivity screening assays like enhanced chemiluminescence technology and nucleic acid testing (ID-NAT).

\section{Material and Methods}

Blood was collected from donors (voluntary and replacement donors) from January, 2019 to December, 2019 with strict donor selection criteria and after taking detailed history and thorough clinical examination to eliminate professional donors. All serum samples were screened for the presence of anti HIV 1 and 2 antibody, anti HCV antibody and HBsAg by chemiluminescence (VITROS ${ }^{\circledR} 3600$ system) and ID-NAT (Porcleix ${ }^{\circledR}$ Panther $®$ System) with internal quality controls performed daily by using both positive and negative controls from the manufacturers. Statistical analysis was made by the Statistical Package for the Social Sciences (SPSS Inc., Chicago, Illinois, USA, version 17.0) for Windows software program and Chi-Square Tests and $p$ value $<0.05$ was considered to be statistically significant. Sensitivity, specificity and diagnostic accuracy of both the screening test modality was also assessed.

\section{Observations}

This study was carried out at Blood and Component Bank of Jawaharlal Nehru Medical College and Hospital, AMU, Aligarh from January 2019 to December 2019. The total number of blood donations was 23074. Majority of the blood donors were young in the 3rd decade of life, 13742 (59.5\%); followed by in 4th decade of life, 4547 (19.7\%) and $2276(9.8 \%)$ in the 6 th decade of life (Table 1$)$.

There were 22303(96.6\%) male donors as compared to $771(3.3 \%)$ female donors, with male-female ratio of 28.9:1, which was statistically significant $(p<0.001)$ (Table 2). Replacement blood donations comprised the major type 
of donation, with 21392 (92.7\%) donations and voluntary donations comprised of $1682(7.2 \%)$ donations, with a statistically significant correlation $(\mathrm{p}<0.05)$ (Table 3$)$.

\begin{tabular}{|c|c|c|}
\hline Age(Years) & No. of Donors & Percentage \\
\hline Oct-20 & 2307 & 9.9 \\
\hline $21-30$ & 13742 & 59.5 \\
\hline $31-40$ & 4547 & 19.7 \\
\hline $41-50$ & 2276 & 9.8 \\
\hline$>50$ & 192 & 0.8 \\
\hline Total & $\mathbf{2 3 0 7 4}$ & $\mathbf{1 0 0}$ \\
\hline
\end{tabular}

Table 1: Distribution of Blood Donors according to Age.

\begin{tabular}{|c|c|c|}
\hline Gender & No. of Donors & Percentage \\
\hline Males & 22303 & 96.6 \\
\hline Females & 771 & 3.3 \\
\hline Total & $\mathbf{2 3 0 7 4}$ & $\mathbf{1 0 0}$ \\
\hline
\end{tabular}

Table 2: Distribution of Blood Donors according to Gender.

\begin{tabular}{|l|c|c|}
\hline Type of Donation & No. of Donors & Percentage \\
\hline Replacement & 21392 & 92.7 \\
\hline Voluntary & 1682 & 7.2 \\
\hline Total & $\mathbf{2 3 0 7 4}$ & $\mathbf{1 0 0}$ \\
\hline
\end{tabular}

Table 3: Distribution of Blood Donors according to the type of Donation.

In our study, 22377(96.9\%) blood donors were nonreactive by chemiluminescence. The seroprevalence of infectious disease HIV was $29(0.1 \%)$, HCV was $160(0.6 \%)$ and HBsAg was 508(2.2\%) by chemiluminescence (Table 4).

\begin{tabular}{|c|c|c|}
\hline Seroprevalence & No. of Donors & Percentage \\
\hline HIV & 29 & 0.1 \\
\hline HCV & 160 & 0.6 \\
\hline HBsAg & 508 & 2.2 \\
\hline Non-Reactive & 22377 & 96.9 \\
\hline Total & $\mathbf{2 3 0 7 4}$ & $\mathbf{1 0 0}$ \\
\hline
\end{tabular}

Table 4: Seroprevalence of HIV, HCV, HBsAg by Chemiluminescence.

The number of non-reactive blood donors in our study decreased slightly by ID-NAT as compared to chemiluminescence, which was 22321(96.7\%). The seroprevalence of various infectious diseases by ID-NAT was $18(0.07 \%)$ of HIV, $137(0.5 \%)$ of $\mathrm{HCV}$ and $598(2.5 \%)$ of
HBsAg (Table 5).

\begin{tabular}{|c|c|c|}
\hline Seroprevalence & No. of Donors & Percentage \\
\hline HIV & 18 & 0.07 \\
\hline HCV & 137 & 0.5 \\
\hline HBsAg & 598 & 2.5 \\
\hline Non-Reactive & 22321 & 96.7 \\
\hline Total & $\mathbf{2 3 0 7 4}$ & $\mathbf{1 0 0}$ \\
\hline
\end{tabular}

Table 5: Seroprevalence of HIV, HCV, HBsAg by ID-NAT.

The sensitivity, specificity and diagnostic accuracy by chemiluminescence of various infectious disease screening was $97.7 \%, 95.5 \%$ and $97.0 \%$ respectively and the sensitivity, specificity and diagnostic accuracy by ID-NAT was $99.2 \%$, $96.7 \%$ and $99.8 \%$ respectively (Table 6 ).

\begin{tabular}{|c|c|c|}
\hline & $\begin{array}{c}\text { Chemiluminescence } \\
\text { (\%) }\end{array}$ & $\begin{array}{c}\text { ID-NAT } \\
\text { (\%) }\end{array}$ \\
\hline Sensitivity & 97.7 & 99.2 \\
\hline Specificity & 95.5 & 96.7 \\
\hline Diagnostic Accuracy & 97 & 99.8 \\
\hline
\end{tabular}

Table 6: Comparative evaluation of Chemiluminescence and ID-NAT screening according to Sensitivity, Specificity and Diagnostic Accuracy.

\section{Discussion}

Technological advancements have led to the development of more sensitive methods to detect various infectious disease markers, e.g., viral specific antigens, antibodies and nucleic acids in order to enhance the safety of blood transfusion $[8,9]$. However, early detection of infection remains elusive goal due to the existing problem of "Window period," false negative results due to the limitation in the screening assays, genetic modifications in viral strains, and laboratory errors [10]. Since ours is a hospital-based blood bank, majority of the blood units are collected from the replacement donors and very few are voluntary donors.

The problem of blood borne infections poses a major threat still in developing countries, to safe blood transfusion due to less number of voluntary donations, non-uniformity of screening policy, use of less sensitive assays for viral screening and high prevalence of the viral diseases like Hepatitis B and C and HIV. In India according to Drugs and Cosmetic act, it is mandatory to screen the blood units for serological markers of HIV, HBV, HCV, syphilis and malaria. The current mandatory screening strategy in the country does not address the problem of critical window period case detection. The period of time from infection to the time of 


\section{Haematology International Journal}

detection of the infection by any given blood screening assay is called window period and with test results and algorithms of pooled and ID-NAT window phase transmission risk models have been developed [11].

Based on the seroprevalence study among blood donors by dual testing strategy using hemiluminescence and NAT testing, our study reveals serious concerns regarding the HIV, HBV, and HCV infections among the blood donors and the safety of the blood supply in our country. Considering the vast population of the country, even low prevalence amounts to large number of infected people.

Molecular virological techniques play a key role in diagnosis and monitoring of treatment for HCV. Because it is difficult to cultivate the virus in cell culture, molecular techniques were instrumental in first identifying HCV, making it one of the first pathogens to be identified by purely molecular methods. Chemiluminescence Immunoassay (CIA) is an antibody test similar to the EIA. For the diagnosis of $\mathrm{HCV}$, the CIA has similar sensitivity and specificity as the third-generation EIA [10]. NAT is considered the 'gold standard' for detecting active HCV replication. HCV NAT is extremely useful in establishing the diagnosis of acute HCV infection, since RNA is detectable as early as 1 week after exposure via needle-stick or blood transfusion, and at least 4-6 weeks prior to seroconversion as demonstrated in a number of transmission settings $[5,9]$. The diagnosis of HCV infection is established with antibody screening followed by NAT for HCV RNA for confirmation as well as for follow-up of patients on treatment $[9,12]$.

Accurate estimates of risk of TTIs are essential for monitoring the safety of blood supply and evaluating the efficacy of the currently employed screening procedures. In India screening for HIV, hepatitis B, and hepatitis C is based on serological testing with recent introduction of NAT testing in few centers. Even after implementing the more sensitive, newest generation of serological tests, a considerable residual risk of transfusion of these viruses' remains [9]. Most populations in resource-limited regions suffer from high prevalence rate of TTIs, and are expected to have more frequent incident cases, as well as more occult carriers. Only countries with a high prevalence and incidence of infection are likely to yield significant number of window period donations. Consequently, NAT screening of TTIs in these populations would be expected to identify more yield cases as compared to the developed world and thus to be more cost effective [8].

China and India are two countries with large populations where the adoption of NAT could have a significant impact on the rate of TTIs. Around the world, more than 53 million units of blood are screened with NAT annually. $100 \%$ of the
USA blood supply is screened with NAT for HIV-1, HCV, HBV and West Nile virus [13]. Although the yield of NAT-only units is modest relative to the yield of serological screening, the infectivity of viremic donations detected by NAT (with or without detectable serological markers) is very high. Hence, the relative impact of NAT screening is arguably greater than that of serological screening, although the existence of seropositive but NAT-negative donations indicates that serological screening must be maintained even with the most sensitive NAT testing performed on individual donations $[14,15]$.

The risk of TTI has declined dramatically in developed countries over the past two decades as a result of a cumulative approach of remarkable improvement in repeat voluntary blood donation and simultaneous testing of blood donors with NAT technology. Marwaha N, et al. and Tiwari AK, et al. have observed NAT yield of $0.034 \%$ ( 1 in 2972 donations) and $0.038 \%$ ( 1 in 2622 donations) respectively [16,17]. The combined yield (seronegative/NAT reactive) for HIV-I, $\mathrm{HCV}$ and $\mathrm{HBV}$ was $0.065 \%$ [9]. Agarwal, et al. have reported combined NAT yield of 1 in $610(0.16 \%)$ donations. We reported HIV seroprevalence of $0.1 \%$ by chemiluminescence and $0.07 \%$ by ID-NAT in a total of 23074 donor screening $[18,19]$.

Considering the course of chronic HBV infection, in the study by Harvey, et al. total of $129(0.27 \%)$ HBV seroyield cases were identified out of which serologic profile of $>90 \%$ cases were consistent with chronic HBV infection [20]. About 32 cases were $\mathrm{HBeAg}$ positive while ID-NAT nonreactive consistent with immune clearance phase while 96 (74\%) were anti-HBe positive HBeAg negative consistent with inactive carrier state. This inactive carrier state may persist indefinitely, in which the prognosis is generally favorable.

The estimated global prevalence of HCV infection is $3 \%$ which translates to over 180 million people worldwide. High sero-prevalence is observed in Asian and African countries, whereas the developed world including North America, northern and western Europe, and Australia have a low prevalence $[10,15]$. In developing countries, the seroprevalence of HCV displays a high range of variability, ranging from $0.9 \%$ in India to higher prevalence from $2.1-6.5 \%$ in many countries $[5,17]$. Our study has shown a seroprevalence of $\mathrm{HCV}$ of $0.6 \%$ by chemiluminescence and $0.5 \%$ by ID-NAT. Egypt has a reported sero-prevalence of about 22\% and is the highest in the world [10].

$\mathrm{HCV}$ is considered an emerging infection in India. Data available is mostly derived from isolated hospital-based studies and blood banks. The estimates thus obtained have been then extrapolated onto the general population. The estimated HCV prevalence at present is $1-1.9 \%$ [2]. Only one 


\section{Haematology International Journal}

systematic study from West Bengal determined a prevalence of $0.87 \%$ [12]. The majority of the studies in blood donors report prevalence from $0.3-1.85 \%$ [15-17]. The differences can be due to different generations of the anti-HCV assays used and differences in the population and practices between different regions of the country.

Though, blood transfusions have not been ever associated with zero risk, many patients need transfusions and the risk reduction through improvement in infectious disease screening is the need of the hour. Many centers have implemented Nucleic Acid Amplification Testing (NAT) for the purpose of blood safety, it is yet to be mandatory regulatory requirement in India. There is a need for implementation of NAT as an innovative approach in blood banks for reducing the window period and identifying the true sero-prevalence and incidence of Transfusion Transmitted Infections (TTIs) (HBV, HIV and HCV). NAT is highly sensitive way to reduce the window period of HIV to 2.93 days, HBV to 10.34 days and HCV to 1.34 days and definitely improves the transfusion safety $[5,9]$. For evidence based implementation of pooled or ID-NAT large sample size studies based in India are needed. Cost effective adoption of NAT by single center testing in a referral laboratory would help reduce the disease burden in a society where early diagnosis and management would lead to overall health benefit to both donors and patients [17].

In India, the scenario is slowly shifting with blood banks gradually introducing NAT to provide safe blood. In a multicentric study from eight blood banks 8 NAT positive cases in 12,224 samples were detected [9]. Marwaha N, et al. [16] has reported a high combined NAT yield of $0.034 \%$ in 23,779 donors, as compared to other developed countries [16]. Out of a total of 18,354 donors tested by ELSIA and IDNAT in a study from North India, 7 were found to be NATpositive for HBV and HCV [17]. Our study showed a HBsAg seroprevalence of $2.2 \%$ by chemiluminescence and $2.5 \%$ by ID-NAT. The studies with high yield of NAT suggest higher prevalence of TTIs in India and thus the need for NAT in blood banks for screening the donations [21,22]. Though, blood transfusion is being used as supportive therapy to save millions of lives all across the globe each year, it is utmost critical that the transfused blood is safe enough to prevent the spread of blood borne infectious diseases.

The purpose of introduction of NAT in blood banks is for providing additional layer of blood safety. By early detection than serology, the window period of HIV, HBV and HCV infections narrows and in addition with NAT, the issues of donor notification and counselling are resolved well as false reactive donations are identified.

\section{Conclusion}

If high sensitivity serological assays are not used, the safety of the blood for transfusion may become a big concern. Apart from stringent measures in donor screening with better donor recruitment, promoting voluntary blood donation, screening of blood and blood products using better ID-NAT than chemiluminescence would detect potentially infectious blood units in all phases of infection and enhance the safety of the blood and blood components for transfusion.

\section{References}

1. (2010) HIV declining in India, New infections reduced by $50 \%$ from $2000-2009$, Sustained focus on prevention required, NACO Press release pp: 679-683.

2. Chandra T, Rizvi SNF, Agarwal D (2014) Decreasing prevalence of transfusion transmitted infection in Indian scenario. Scientific World J 17(9): 17-19.

3. Kumar R, Gupta S, Kaur A, Gupta M (2015) Individual donor-nucleic acid testing for human immunodeficiency virus-1, hepatitis $C$ virus and hepatitis $B$ virus and its role in blood safety. Asian J Transfus Sci 9(2): 199-202.

4. Brennan TA, Shrank WH (2014) Costs of New Treatments for Hepatitis C Infection-Reply. JAMA 312: 2168-2169.

5. Ismail N, Fish GE, Smith MB (2004) Laboratory evaluation of a fully automated chemiluminescence immunoassay for rapid detection of HBsAg, antibodies to HBsAg, and antibodies to hepatitis C virus. J Clin Microbiol 42: 610617.

6. Grabarczyk P, Van Drimmelen H, Kopacz A (2013) Headto-head comparison of two transcription-mediated amplification assay versions for detection of hepatitis B virus, hepatitis $C$ virus, and human immunodeficiency virus type 1 in blood donors. Transfusion 53(10): 25122524.

7. (2010) HIV AIDS in South-East Asia Region: progress report 2010, WHO Regional Office for South-East Asia.

8. Ghosh K, Mishra K (2017) Nucleic acid amplification testing in Indian blood banks: A review with perspectives. Ind J Pathol Microbiol 60(3): 313-318.

9. Chandra T, Rizvi SNF, Agarwal D (2016) Nucleic acid testing in blood donors in Northern India- A single centre Experience. Ind J of Contemporary Med Res 3(6): 1818-1821.

10. Yang Z, Xu L, Liu L (2013) Routine screening of blood donations at Qingdao central blood bank, China, for hepatitis B virus (HBV) DNA with a real-time, multiplex nucleic acid test for HBV, hepatitis $\mathrm{C}$ virus, and human immunodeficiency virus Types 1 and 2. Transfusion 53: 2538-2544. 
11. Weusten JJ, Vermeulen $M$, van Drimmelen $H$, Lelie $N$ (2011) Refinement of a viral transmission risk model for blood donations in seroconversion window phase screened by nucleic acid testing in different pool sizes and repeat test algorithms. Transfusion 51(1): 203-215.

12. Nayak NC, Jain D, Vasdev N, Gulwani H, Saigal S, et al. (2012) Etiologic types of end-stage chronic liver disease in adults: Analysis of prevalence and their temporal changes from a study on native liver explants. Eur J Gastroenterol Hepatol 24: 1199-2208.

13. Baylis SA, Wallace $P$, McCulloch E, Niesters HG, Nübling CM (2019) Standardization of nucleic acid tests: The approach of the World Health Organization. J Clin Microbiol 57: 1056-1058.

14. Makroo RN, Chowdhry M, Bhatia A, Antony M (2015) Evaluation of the Procleix Ultrio Plus ID NAT assay for detection of HIV 1, HBV and HCV in blood donors. Asian J Transfus Sci 9: 29-30.

15. Chigurupati P, Murthy KS (2015) Automated nucleic acid amplification testing in blood banks: An additional layer of blood safety. Asian J Transfus Sci 9: 9-11.

16. Marwaha N, Hans R, Sachdev S, Sharma S, Sharma RR (2014) Results of nucleic acid testing in blood donors A study from a tertiary healthcare centre in North India. Vox Sang 107(1): 247-249.
17. Tiwari AK, Dara RC, Arora D, Aggarwal G, Rawat G, et al. (2017) Comparison of two algorithms to confirm and discriminate samples initially reactive for nucleic acid amplification tests. Asian J Transfus Sci 11(2): 140-146.

18. Agarwal N, Chatterjee K, Coshic P, Borgohain M (2013) Nucleic acid testing for blood banks: An experience from a tertiary care centre in New Delhi, India. Transfus Apher Sci 49(3): 482-484.

19. Chaurasia R, Zaman S, Das B, Chatterjee K (2014) Screening donated blood for transfusion transmitted infections by serology along with NAT and response rate to notification of reactive results: an Indian experience. J Blood Trans 105: 6-12.

20. Doda V, Arora S, Kirtania T (2014) Serological characterization of occult hepatitis $B$ virus infection among blood donors in India. Transfus Apher Sci 51(2): 162-167.

21. Bhargava A, Pathak N, Varshney S, Shrivastava M, Mishra PK (2014) Molecular detection of window phase hepatitis $\mathrm{C}$ virus infection in voluntary blood donors and health care workers in a cohort from Central India. Indian J Community Med 39(1): 51-52.

22. Hans R, Marwaha N (2014) Nucleic acid testing-benefits and constraints. Asian J Trans Sci 8(1): 2-3. 